\title{
FREE ACTIONS ON $S^{n} \times S^{n}\left({ }^{1}\right)$
}

\author{
BY \\ GENE LEWIS
}

0. Introduction. Conner [4], Heller [5], Mann [8], Mann and Su [9], and Su [12] have investigated free actions on a product of spheres. The best result is due to Heller [5]:

Proposition O. Let $n, m \geqq 1$. Then $Z_{p} \oplus Z_{p} \oplus Z_{p}$ cannot act freely on $S^{n} \times S^{m}$.

To my knowledge, no one has considered actions of nonabelian groups. I will prove:

THeOREM A. Let $n$ be odd, $p$ an odd prime, $p \nmid n+1$. Then any p-group acting freely on $S^{n} \times S^{n}$ is abelian.

THEOREM B. Let $n \equiv 1$ (4). Then any 2-group acting freely, preserving orientation, on $S^{n} \times S^{n}$, is abelian.

The method of proof is via a Gysinoid sequence (\$2), which holds whenever a finite group acts freely on a manifold $M$ with three nonzero homology groups. The most useful cases are (1) $M=S^{n} \times S^{n}$ and (2) $M=$ a surface.

We give a short proof of Mazur's theorem that $H^{\mathrm{i}}(G, Z) \neq 0$ for infinitely many $i>0$, and of the fact that $Z_{p}^{3}$ cannot act freely on $S^{n} \times S^{n}$, preserving orientation. Finally, we discuss the Conjecture: If $G$ acts freely on $S^{n} \times S^{n}, Z_{2} \oplus Z_{2} \subset G$, then $Z_{2} \oplus Z_{2} \cap Z(G) \neq 1$. We prove this for some special cases.

1. Topological preliminaries. The following discussion is necessary in order to prove Theorems A and B for arbitrary continuous actions.

In $E^{n+1}$, let $e_{0}=(1,0, \ldots, 0), \ldots, e_{n}=(0,0, \ldots, 1)$ be a standard basis, $\Delta^{n}$ $=$ convex hull of $\left\{e_{0}, \ldots, e_{n}\right\}=$ standard $n$-simplex. If $Y$ is a topological space (assumed arcwise connected), and $F$ is a system of local coefficients, a la Steenrod, on $Y$, we define $H^{*}(Y, F)=$ singular cohomology of $Y$ with local coefficients $F$, as follows:

Let $\Sigma_{i}(Y)=$ all $i$-simplices of $Y$. $\Gamma_{s}^{i}(Y, F)=\left\{f: \Sigma_{i}(Y) \rightarrow F \mid f(\sigma) \in F_{\sigma(0)}\right.$, for all $\left.\sigma \in \Sigma_{i}(Y)\right\}$. Define $\delta: \Gamma_{s}^{i}(Y, F) \rightarrow \Gamma_{s}^{i+1}(Y, F)$ by

$$
\delta f(\sigma)=\sum_{i=1}^{n}(-1)^{i} f\left(\sigma^{i}\right)+\alpha^{*} f\left(\sigma^{0}\right),
$$

Received by the editors October 21, 1966.

(1) This work was done when the author was an NSF Graduate Fellow. 
where $\sigma^{i}=i$ th face of $\sigma \in \Sigma_{i+1}(Y)$, and $\alpha=\sigma \mid\left(e_{0} e_{1}\right)$. [The point is that $\sigma^{i}\left(e_{0}\right)=\sigma\left(e_{0}\right)$ for $0<i \leqq n$, but $\sigma^{0}\left(e_{0}\right)=\sigma\left(e_{1}\right)$.] Then $\delta^{2}=0$, and we set $H_{s}^{*}(Y, F)=H^{*}\left(\Gamma_{s}(Y, F)\right)$.

Let $A=G$-module, $Y=$ a topological space on which $G$ acts freely. Then $Y \stackrel{\pi}{\rightarrow} Y / G$ is a finite covering space. We form $\tilde{A}=Y \times{ }_{G} A=Y \times A / \sim$, where $(y, a) \sim(g y, g a) . \pi$ induces $\tilde{\pi}: \tilde{A} \rightarrow Y / A$. Claim: $\tilde{A}$ is a local coefficient system over $Y / G$. For if $x_{0}, x_{1} \in Y / G, \alpha$ a path from $x_{0}$ to $x_{1}$, choose $y_{1} \in Y$ such that $\pi\left(y_{1}\right)=x_{1}$. By properties of covering spaces, there exists a unique $\tilde{\alpha}:[0,1] \rightarrow Y$, with $\tilde{\alpha}(1)=y_{1}$, $\pi \tilde{\alpha}=\alpha$. Set $y_{0}=\tilde{\alpha}(0)$. This is uniquely determined by the homotopy class of $\alpha$ rel $\left\{x_{0}, x_{1}\right\}$. If $u \in \tilde{A}_{x_{1}}, u=\left[\left(y_{1}, a\right)\right]$, set $\alpha^{*} u=\left[\left(y_{0}, a\right)\right]$. Taking $g y_{1}$ in place of $y_{1}$ yields $g \tilde{\alpha}$ in place of $\tilde{\alpha}$, by uniqueness, hence gives $g y_{0}$ for $y_{0}$. Thus $\alpha^{*}: \tilde{A}_{x_{1}} \rightarrow \tilde{A}_{x_{0}}$ is well defined, and clearly $\left(\alpha_{1} \circ \alpha_{2}\right)^{*}=\alpha_{2}^{*} \circ \alpha_{1}^{*}$. Thus $\tilde{A}$ is a local coefficient system. The following is well known:

Lemma 1.1. $\operatorname{Hom}_{G}(S(Y), A) \approx \Gamma_{S}(Y / G, \tilde{A})$, as chain complexes.

Proof. We will define reciprocal isomorphisms $\phi$ (left to right), and $\psi$ (right to left). If $f \in \operatorname{Hom}_{G}\left(S_{i}(Y), A\right)$, let $\bar{\sigma} \in \Sigma_{i}(Y / G)$. Given $y$, such that $\pi(y)=\bar{\sigma}(0)$, there exists a unique lifting $\sigma_{y}: \Delta^{i} \rightarrow Y$ such that $\sigma_{y}(0)=y$. By uniqueness, $g \sigma_{y}=\sigma_{g y}$. Thus the pair $\left(y, f\left(\sigma_{y}\right)\right)$ defines an element of $\tilde{A}_{\tilde{\sigma}(0)}$, independent of choice of $y$.

Set $\phi(f)(\bar{\sigma})=\left[\left(y, f\left(\sigma_{y}\right)\right]\right.$. Given $\gamma \in \Gamma_{s}^{i}(Y / G, \tilde{A})$, we wish to define a $G$-morphism $\psi(\gamma): S_{i}(Y) \rightarrow A$. As $S_{i}(Y)$ is $G$-free, it suffices to define $\psi(\gamma)$ on $\Sigma_{i}(Y)$. Let $\sigma \in \Sigma_{i}(Y), \bar{\sigma}=\pi(\sigma)$. Then define $\psi(\gamma)(\sigma)$ by $\gamma(\bar{\sigma})=[(\sigma(0), \psi(\gamma)(\sigma))] . \psi(\gamma)$ is clearly a $G$-morphism. $\phi \psi=1, \psi \phi=1$ are obvious. We leave to the reader the simple-minded task of checking that $\psi$ and $\phi$ are chain maps. Q.E.D.

LEMMA 1.2. Let $X$ be an arcwise connected, paracompact, topological n-manifold. If $F$ is any system of local coefficients on $X$, then $H_{S}^{i}(X, F)=0$, for $i>n$.

Proof. Such a well-known result needs no proof.

Lemma 1.3 (Heller). Let $Y$ be an n-manifold, $G$ a finite group acting freely on $Y$. $S(Y)=$ singular complex of $Y$, and $B_{n}(Y)=\operatorname{Im}\left\{\partial: S_{n+1}(Y) \rightarrow S_{n}(Y)\right\}$. Then $B_{n}$ is G-projective.

Proof. $B_{n}$ is $G$-projective if and only if for every $G$-module $A$, $\operatorname{Ext}^{i}\left(B_{n}, A\right)=0$, $i>0 . \operatorname{Ext}_{G}^{i}\left(B_{n}, A\right) \approx H^{n+1+i}\left(\operatorname{Hom}_{G}(S(Y), A)\right)$, since $\cdots \rightarrow S_{n+2} \rightarrow S_{n+1} \rightarrow B_{n} \rightarrow 0$ is a free $G$-resolution of $B_{n}$. By Lemma 1.1 , this is $\approx$ to $H_{S}^{n+1+i}(Y / G, \tilde{A})$, and by Lemma 1.2, the latter is zero. Q.E.D.

COROLlaRY 1.4. Under the hypotheses of Lemma 1.3, we have

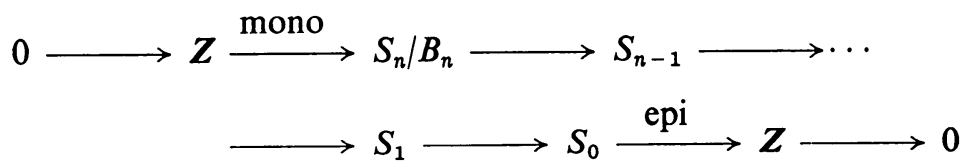

exact at $S_{0}$, with $S_{n} / B_{n} G$-cohomologically trivial. 
Application:

Proposition 1.5 (MAZUR). If $G$ is a finite group, then $H^{i}(G, Z) \neq 0$, for infinitely many $i>0$.

Proof. Imbed $G \subset U(n)$, as a subgroup, suitable $n$. $G$ acts freely on $U(n)$ by left multiplication. As $\pi_{0}(U(n))=0$, each $g \in G$ has $L_{g} \simeq 1$. Hence $G$ acts trivially on $H^{*}(U(n), Z)=E\left(u_{1}\right) \otimes E\left(u_{3}\right) \otimes \cdots \otimes E\left(u_{2 n-1}\right)$ (torsion free). By Corollary 1.4, $U(n)$ has a finite $G$-complex

$$
C: 0 \rightarrow Z \rightarrow C_{n^{2}} \rightarrow \cdots \rightarrow C_{0} \rightarrow Z \rightarrow 0
$$

with all $C_{i}$ cohomologically trivial. The cohomology of $C^{*}=\operatorname{Hom}(C, Z)$ are either 0 , or finite sums of $Z$, trivial $G$-action. The result follows by splicing $C^{*}$ into short exact sequences, and applying Tate cohomology and dimension-shifting. Q.E.D.

2. The exact sequence, and applications. Let $Y$ be an $n$-manifold, $G$ a finite group acting freely on $Y$. Suppose $Y$ has precisely three nonzero homology groups, in dimensions $0, l$, and $n: H_{0}(Y)=Z, H_{l}(Y) \neq 0, H_{n}(Y)=Z^{\prime}$. [Note that these are $G$-modules. If $G$ has no subgroup of index $2, Z^{\prime}=Z$ with trivial action. In any case, $G$ acts trivially on $H_{0}(Y)$.]

Proposition 2.1. The sequence (2.2) is exact $(i \in Z)$ :

$$
\begin{aligned}
\cdots \rightarrow \hat{H}^{i+n-l}\left(G, Z^{\prime}\right) & \rightarrow \hat{H}^{i-l-1}(G, Z) \rightarrow \hat{H}^{i}\left(G, H_{l}(Y)\right) \\
& \rightarrow \hat{H}^{i+1+n-l}\left(G, Z^{\prime}\right) \rightarrow \cdots
\end{aligned}
$$

Proof. By Corollary 1.4, $Y$ has a finite $G$-complex $C$ :

$$
0 \rightarrow Z^{\prime} \rightarrow C_{n} \rightarrow \cdots \rightarrow C_{l} \rightarrow \cdots \rightarrow C_{0} \rightarrow Z \rightarrow 0,
$$

exact except at $C_{l}$, where the homology is $H_{l}(Y)$. Each $C_{i}$ is cohomologically trivial. Splice

$$
\begin{aligned}
& 0 \rightarrow Z^{\prime} \rightarrow C_{n} \rightarrow \cdots \rightarrow C_{l+1} \rightarrow B_{l} \rightarrow 0 \quad \text { exact, } \\
& 0 \rightarrow Z_{l} \rightarrow C_{l} \rightarrow \cdots \rightarrow C_{0} \rightarrow Z \rightarrow 0 \quad \text { exact. }
\end{aligned}
$$

$0 \rightarrow B_{l} \rightarrow Z_{l} \rightarrow H_{l}(Y) \rightarrow 0$. The long exact sequence implies

$$
\cdots \rightarrow \hat{H}^{i}\left(G, B_{l}\right) \rightarrow \hat{H}^{i}\left(G, Z_{l}\right) \rightarrow \hat{H}^{i}\left(G, H_{l}(Y)\right) \rightarrow \hat{H}^{i+1}\left(G, B_{l}\right) \rightarrow \cdots,
$$

$\left(^{*}\right),\left(^{* *}\right)$ imply $\hat{H}^{i}\left(G, B_{l}\right) \approx \hat{H}^{i+n-l}(G, Z)^{\prime}, \hat{H}^{i}\left(G, Z_{l}\right) \approx \hat{H}^{i-l-1}(G, Z)$. Substituting these values in $(\star)$ gives the result. Q.E.D.

COROLlaRY 2.3. In the above situation, if $G$ preserves orientation, and if $H_{l}(Y)$ is cohomologically trivial, then $G$ is periodic of period $n+1$.

Proof. For then $Z^{\prime}=Z$, and $\hat{H}^{n+1}(G, Z) \approx \hat{H}^{0}(G, Z) \approx Z / g Z, g=|G|$. By [3, Chapter XII, $\S 11]$, done. (This implies $n$ is odd, $\Rightarrow n=3$.) 
REMARK 1. Poincaré duality and universal coefficient theorem imply either $n=2 l$ or $n=3, l=1$.

REMARK 2. If $Y$ is a cell complex and if $G$ acts freely, cellularly, then the cells afford a finite $G$-complex for $Y$. Hence the argument goes through and Proposition 2.1 holds in this case also.

Case 1. $Y=$ a closed compact surface. $n=2, l=1$. Therefore (2.2) is

$$
\cdots \rightarrow \hat{H}^{i}(G, Z) \rightarrow \hat{H}^{i-3}(G, Z) \rightarrow \hat{H}^{i-1}\left(G, H_{1}(Y)\right) \rightarrow \hat{H}^{i+1}(G, Z) \rightarrow \cdots
$$

Suppose $G$ preserves orientation. Then $Z^{\prime}=Z$. Set $i=1$. As

$$
H^{2}(G, Z) \approx \operatorname{Hom}(G /[G, G], Q / Z)=(G /[G, G])^{\wedge} \quad(\approx G /[G, G]),
$$

and $H^{1}(G, Z)=0, \hat{H}^{-i}(G, Z) \approx \hat{H}^{i}(G, Z)$, we get $\hat{H}^{-2}(G, Z) \approx H_{1}(G, Z) \approx G /[G, G]$,

$$
0 \rightarrow G /[G, G] \rightarrow \hat{H}^{0}\left(G, H_{1}(Y)\right) \rightarrow(G /[G, G])^{\wedge} \rightarrow 0 .
$$

Sad to say, this striking result seems almost useless. The sequence is not split in general. If $Y=S^{1} \times S^{1}=$ torus, then $H_{1}(Y)=Z \oplus Z$ and using the Lefschetz formula it is easy to see that $G$ acts trivially on $Z \oplus Z$ (see below). Thus

$$
\hat{H}^{0}(G, Z \oplus Z) \approx Z_{g} \oplus Z_{g}, \quad g=|G| .
$$

This implies $|G /[G, G]|=g$, implying $[G, G]=1$, i.e. $G$ is abelian. Thus our sequence (2.4) is just $0 \rightarrow G \rightarrow Z_{g} \oplus Z_{g} \rightarrow G \rightarrow 0$. Hence $G=$ sum of two cyclic groups. If $G=Z_{n} \oplus Z_{n}$ with obvious action on $S^{1} \times S^{1}$, then $g=n^{2}$ and $0 \rightarrow Z_{n} \oplus Z_{n} \rightarrow$ $Z_{n^{2}} \oplus Z_{n^{2}} \rightarrow Z_{n} \oplus Z_{n} \rightarrow 0$ is not split. We have proved:

Proposition 2.5. If $G$ acts freely, preserving orientation, on the torus, then $G$ is the sum of two cyclic groups.

REMARK 1. This is an easy exercise in covering spaces. That is the way L. E. J. Brouwer first proved it in 1919 [2].

REMARK 2. It is simple to prove that any finite group acts freely on a 2-sphere with handles $Y$ (see [1]). In case $G$ preserves orientation, if $r=$ the number of handles, then $H_{1}(Y) \approx Z^{2 r}, r \equiv 1(g)$, by Hurwitz formula, and $\operatorname{tr} g=2$ for all $g \in G-\{1\}$, by Lefschetz formula.

REMARK 3. Using the Swan spectral sequence [13], one can explicitate the maps in (2.2) via the multiplication structure. Thus, in the case of a surface, the map $\hat{H}^{i}(G, Z) \rightarrow \hat{H}^{i-3}(G, Z)$ is cup product with a class $\sigma \in \hat{H}^{-3}(G, Z)$. As we have no application for the product structure, we omit details.

REMARK 4. In case of a surface, (2.2) was noted (independently) by Kawada and Tate [6].

Case 2. $Y=S^{n} \times S^{n}$. (i) Suppose $n$ odd. Claim: $G$ acts trivially on $H_{n}(Y)=Z \oplus Z$ (if $G$ preserves orientation). For, if $g \in G, g \neq 1,0=\operatorname{tr}_{0} g-\operatorname{tr}_{n} g+\operatorname{tr}_{2 n} g=1-\operatorname{tr}_{n} g+1$, by Lefschetz formula, implying $\operatorname{tr}_{n} g=2$. $g^{r}=1$, some $r$ implies det $g= \pm 1$. Suppose det $g=1$. Then $g$ has characteristic polynomial $g^{2}-2 g+1=(g-1)^{2}=0$, implying 
$g$ has a nonzero fixed point, $\phi=(a, b)$, say. We assume g.c.d. $(a, b)=1$. If $a c+b d=1$, then $\psi=(c, d)$ is a complement for $\phi$. In the $\{\psi, \phi\}$ basis,

$$
g \sim\left(\begin{array}{ll}
\alpha & \beta \\
0 & 1
\end{array}\right)
$$

$\operatorname{tr} g=2 \Rightarrow \alpha=1 . g^{r}=1 \Rightarrow r \beta=0 \Rightarrow \beta=0$. That is,

$$
g \sim\left(\begin{array}{ll}
1 & 0 \\
0 & 1
\end{array}\right)
$$

i.e. $g$ acts trivially.

Thus det $g=1$ implies $g$ acts trivially. If $\operatorname{det} g=-1$, then $\operatorname{det} g^{2}=1 \Rightarrow g^{2}=1$ (as transformation). The characteristic polynomial is $g^{2}-2 g-1=0, g^{2}=1 \Rightarrow g=0$. Contradiction. Hence det $g=1$ always, so $G$ acts trivially as asserted.

(2.2) now becomes

$$
\begin{aligned}
\cdots \rightarrow H^{i}(G, Z \oplus Z) & \rightarrow H^{i+n+1}(G, Z) \rightarrow H^{i-n}(G, Z) \\
& \rightarrow H^{i+1}(G, Z \oplus Z) \rightarrow \cdots
\end{aligned}
$$

CoRollary 2.7. Suppose $n$ odd, and $G$ acts freely on $S^{n} \times S^{n}$, p.o. (preserving orientation). Then

$$
0 \rightarrow H^{n}(G, Z) \rightarrow H^{n+1}(G, Z) \rightarrow Z_{g} \oplus Z_{g} \rightarrow H^{n+1}(G, Z) \rightarrow H^{n}(G, Z) \rightarrow 0
$$

is exact. (Set $i=0$ in (2.6). We have identified $H^{-m}(G, Z) \approx H^{m}(G, Z)$.)

Proposition 2.8 (CONNER). $Z_{p} \oplus Z_{p} \oplus Z_{p}$ cannot act freely, p.o. on $S^{n} \times S^{n}$.

Proof. We will see below that $1, Z_{2}, Z_{4}, Z_{2} \oplus Z_{2}$ are the only groups acting freely on $S^{n} \times S^{n}$, for $n$ even. If $n$ odd, apply Corollary 2.7. $g=p^{3}$. We know that $p H^{*}\left(Z_{p} \oplus Z_{p} \oplus Z_{p}, Z\right)=0$ in positive dimensions implies we have $Z_{p}^{a} \rightarrow Z_{p^{3}} \oplus$ $Z_{p^{3}} \rightarrow Z_{p}^{b}$, exact, some $a, b$. Contradiction. Q.E.D.

THEOREM A. If $n$ odd, $p$ odd prime, $p \nmid n+1$ and if $G$ is a p-group acting freely on $S^{n} \times S^{n}$, then $G$ is abelian.

Proof. It suffices to show that $G$ contains no minimal nonabelian $p$-groups. Rédei [11] found these to be of two sorts ( $p$ odd).

Type 1. $G=\left(A, B: A^{p^{u}}=B^{p^{v}}=1, B^{A}=B^{1+p^{v-1}}\right), u \geqq 1, v \geqq 2$.

Type 2. $G=\left(A, B: A^{p u}=B^{p^{v}}=C^{p}=1, A C=C A, B C=C B, B^{A}=B C\right), u \geqq v \geqq 1$.

A group $G$ of Type 1 is a split extension: $1 \rightarrow Z_{p^{v}} \rightarrow G \rightarrow Z_{p^{u}} \rightarrow 1\left(^{*}\right)$. Apply [7, Proposition 5.1], with $r=p^{v}, s=p^{u}, t=1+p^{v-1}, a=(n+1) / 2$. Corollary 2.7 implies $\alpha_{n+1}=g \alpha_{n}$. Here $g=p^{u+v}, n+1=2 a, n=2 a-1$. By [7, Proposition 5.1]

$$
H^{2 a}(G, Z) \approx Z_{s} \oplus \sum_{1 \leqq i<a} Z_{q_{i}} \oplus Z_{h_{a}}, \quad H^{2 a-1}(G, Z) \approx \sum_{1 \leqq i \leqq a-1} Z_{q_{i}},
$$


where $h_{i}=\left(t^{i}-1, r\right), k_{i}=\left(\sum_{j=0}^{s-1} t^{i j}, r\right), q_{i}=h_{i} k_{i} / r$. Thus $\alpha_{2 a}=s h_{a} \alpha_{2 a-1} \Rightarrow s h_{a}=p^{u+v}$, or $h_{a}=p^{v}$. But calculation shows

$$
\begin{aligned}
& h_{i}=p^{v-1} \quad p \nmid i, \\
& =p^{v} \quad p \mid i .
\end{aligned}
$$

As $p \nmid a$, we have a contradiction. Hence no Type 1 group can act freely on $S^{n} \times S^{n}$ if $p \nmid n+1$.

If $G$ is a Type 2 group acting freely on $S^{n} \times S^{n}$, by Proposition 2.8, $Z_{p} \oplus Z_{p}$ $\oplus Z_{p} \nsubseteq G$. If $u>1$, then $A^{p k-1} \neq 1 \in Z(G)$, so that the group $\left\langle C, B, A^{p k-1}\right\rangle$ is abelian and contains a subgroup $\approx Z_{p} \oplus Z_{p} \oplus Z_{p}$. Contradiction. Hence $u=1, \Rightarrow v=1 \Rightarrow G$ is the group of order $p^{3}$ treated in [7, Section 6]. By [7, Corollary 6.27] (with $n+1$ in place of $n$ ), we see $p H^{n+1}(G, Z)=0 . g=p^{3}$, so Corollary 2.7 implies $Z_{p}^{a} \rightarrow Z_{p^{3}} \oplus$ $Z_{p^{3}} \rightarrow Z_{p}^{a}$ exact. Contradiction. Thus no Type 2 group can act freely on $S^{n} \times S^{n}$ if $p \nmid n+1$. This proves Theorem A. Q.E.D.

Proposition 2.9. Suppose $n$ odd, $G$ acting freely, p.o., on $S^{n} \times S^{n}$. If $G$ is periodic, then period $(G) \mid n+1$.

Proof. Corollary 2.7 implies that $\alpha_{n+1}=\alpha_{n} g$. As $\alpha_{n}=1$, this implies $\alpha_{n+1}=g$. With $i=n$ in (2.6) we get: $0 \rightarrow H^{0}(G, Z) \rightarrow H^{n+1}(G, Z) \oplus H^{n+1}(G, Z)$. As $H^{0}(G, Z) \approx Z_{g}$, this means that $H^{n+1}(G, Z) \approx Z_{g} \Rightarrow G$ is periodic, and $p d(G) \mid n+1$ (by [3, Chapter XII, Proposition 11.1]).

THEOREM B. Let $n \equiv 1$ (4). Then a 2-group acting freely, p.o. on $S^{n} \times S^{n}$ is abelian.

Proof. Rédei found three kinds of minimal nonabelian 2-groups: Type 1, Type 2 (as above) and the quaternion group $Q$ of order 8 . Let $G$ be our 2-group. If $Q \subset G$, then as period $Q=4$, Proposition 2.9 implies $4 \mid n+1$, or $n \equiv-1$ (4). Contradiction. Hence $Q \nsubseteq G$. A 2-group of Type 2, not containing $Z_{2} \oplus Z_{2} \oplus Z_{2}$, must be dihedral of order $8, D$. A glance at Even's result [7, (4.0)], shows that $2 H^{n+1}(D, Z)=0$, so Corollary 2.7 again gives a contradiction, implying $D \nsubseteq G$. If a Type 1 group $\supset G$, then as before, $h_{a}=2^{v} . a=(n+1) / 2$. But $4 \nmid n+1$ implies $a$ is odd $\Rightarrow h_{a}=2^{v-1}$. Contradiction. Hence $G$ must be abelian. Q.E.D.

It is well known that if $G$ acts freely on $S^{n}, n$ odd (necessarily p.o., by Lefschetz formula), then $G$ is periodic, and $p d(G) \mid n+1$. This shows that if $G$ acts freely on $S^{n}, S^{m}$ such that g.c.d. $(n+1, m+1)=2$, then $p d(G)=2$ implies $G$ cyclic, [since $\hat{H}^{0}(G, Z) \approx \hat{H}^{2}(G, Z)$ says $\left.Z_{g} \cong G /[G, G] \Rightarrow[G, G]=1, Z_{g} \cong G\right]$.

CONJECTURE 1. If $G$ acts freely, p.o., on $S^{n} \times S^{n}$, and $S^{m} \times S^{m}$, with $(n+1, m+1)$ $=2$, then $G$ is abelian (therefore the sum of two cyclic groups, by Proposition 2.8).

I have only been able to prove the anemic

Proposition 2.10. If $G$ acts freely, p.o. on $S^{n} \times S^{n}$ and $S^{n+2} \times S^{n+2}$, ( $n$ odd), then $G$ is abelian, hence the sum of two cyclic groups. 
Proof. The jumps in (2.6) are $\cdots+(n+1),+(n+1),-(2 n+1),+(n+1)$, $+(n+1) \cdots$. Writing indices in place of the groups in our sequences, we get $1, n+2,-n+1,(2)^{2}, n+3,-n+2,(3)^{2}, n+4,-n+3, \ldots,-3,(n-2)^{2}, 2 n-1,-2$, $(n-1)^{2}, 2 n,-1$. Recall $\alpha_{1}=\alpha_{-1}=1$. Thus for $n=3$, get $(\cdot) \alpha_{6}=\alpha_{2} \alpha_{5}$. For $n \geqq 5$

$$
\alpha_{2} \alpha_{4} \cdots \alpha_{n-1} \alpha_{n+2} \cdots \alpha_{2 n-1}=\alpha_{3} \alpha_{5} \cdots \alpha_{n-2} \alpha_{n+3} \cdots \alpha_{2 n} .
$$

Corollary 2.7 implies (:) $\alpha_{n+1}=g \alpha_{n}$. $-1,(n)^{2}, 2 n+1,0,(n+1)^{2}, 2 n+2,1$ gives (:) $\alpha_{2 n+2}=g \alpha_{2 n+1}$. (i) for $n$ and $n+2$ yields

$$
\frac{\alpha_{2} \alpha_{4} \cdots \alpha_{n+1} \alpha_{n+3} \cdots \alpha_{2 n+1} \alpha_{2 n+3}}{\alpha_{2} \alpha_{4} \cdots \alpha_{n-1} \alpha_{n+2} \cdots \alpha_{2 n-1}}=\frac{\alpha_{3} \cdots \alpha_{n} \alpha_{n+5} \cdots \alpha_{2 n+4}}{\alpha_{3} \cdots \alpha_{n-2} \alpha_{n+3} \cdots \alpha_{2 n}} \quad(n \geqq 3)
$$

implying

$$
\frac{\alpha_{n+1} \alpha_{2 n+1} \alpha_{2 n+3}}{\alpha_{n+2}}=\frac{\alpha_{n} \alpha_{2 n+2} \alpha_{2 n+4}}{\alpha_{n+3}} .
$$

For $n+2$, equation (:) implies $\alpha_{n+3}=g \alpha_{n+2}$. Therefore $g^{2} \alpha_{2 n+1} \alpha_{2 n+3}=\alpha_{2 n+2} \alpha_{2 n+4}$, implying $g \alpha_{2 n+3}=\alpha_{2 n+4}$. Consider

$$
\begin{array}{r}
0 \rightarrow H^{n+2}(G, Z \oplus Z) \rightarrow H^{2 n+3}(G, Z) \rightarrow H^{2}(G, Z) \\
\rightarrow H^{n+3}(G, Z \oplus Z) \rightarrow H^{2 n+4}(G, Z), \\
\searrow_{Y}^{\nearrow} \searrow_{0}
\end{array}
$$

$\alpha_{n+2}^{2} \alpha_{2}|Y|=\alpha_{2 n+3} \alpha_{n+3}^{2}=\alpha_{2 n+3} g^{2} \alpha_{n+2}^{2}, \quad$ or $\quad \alpha_{2}|Y|=g^{2} \alpha_{2 n+3}=g \alpha_{2 n+4}, \quad$ or $\alpha_{2} / g$ $=\alpha_{2 n+4} /|Y| \geqq 1$. Hence $\alpha_{2} \geqq g$. But $\alpha_{2}=|G /[G, G]| \leqq g \Rightarrow \alpha_{2}=g \Rightarrow G$ abelian, as desired. Q.E.D.

Proposition 2.11. If $G$ acts freely on $S^{n}$, $n$ odd, and g.c.d. $(g, n+1)=1$ or 2 , then $G$ is abelian.

Proof. We know $G$ periodic, $p d(G) \mid n+1, G_{p}=$ cyclic, ( $p$ odd), cyclic or generalized quaternion, $(p=2)$ [3, Chapter XII].

Case 1. $g=|G|$ is odd: By Swan's formula for $p$-periods [7, Theorem 3.1], as now $(g, n+1)=1$, and $p d(G)=1 . c . m .2\left|N\left(G_{p}\right): C\left(G_{p}\right)\right| \mid n+1$, we must have $N\left(G_{p}\right)=C\left(G_{p}\right)$, for all prime $p \mid g . G$ is a Z-group, therefore by [15] is of form $1 \rightarrow Z_{r} \rightarrow G \rightarrow Z_{s} \rightarrow 1(r, s)=1$, and therefore, split, by Schur-Zassenhaus. If $p \mid r$, then $\left(Z_{r}\right)_{p}=G_{p}$ is characteristic in $Z_{r}$, and hence normal in $G$. That is, $N\left(G_{p}\right)=G$ implies $G_{p} \subset Z(G)$, so $G$ is cyclic.

Case 2. $g$ even: Here $(g, n+1)=2$ (by hypothesis). As generalized quaternion groups have period 4, [3, Chapter XII, Section 11] we must have $G_{2}=$ cyclic. So $G$ is a $Z$-group such that every subgroup of odd order is cyclic.

Much as in Case 1, it follows that $G$ is cyclic (use Milnor's Theorem: If a group acts freely on $S^{n}$, then every involution lies in the center [10]). Q.E.D. 
REMARK. Milnor's Theorem cannot be dispensed with. For if, say, $\Sigma_{3}$ acted freely on $S^{3}$, the hypotheses of Proposition 2.11 would hold: $(6,4)=2$.

Proposition 2.12. If $n$ is even, $>0$, only 1 and $Z_{2}$ act freely, p.o. on $S^{n} \times S^{n}$; only $1, Z_{2}, Z_{2} \oplus Z_{2}, Z_{4}$ act freely on $S^{n} \times S^{n}$.

Proof. Suppose $G$ acts freely, p.o. If $g \in G-\{1\}$, then $\operatorname{tr}_{0} g+\operatorname{tr}_{n} g+\operatorname{tr}_{2 n} g$ $=2+\operatorname{tr}_{n} g=0$ or $\operatorname{tr}_{n} g=-2$, on $H_{n}\left(S^{n} \times S^{n}\right)=Z \oplus Z$. If $g^{p}=1, p$ odd, then $\operatorname{det} g=1 \Rightarrow$ characteristic polynomial is $g^{2}+2 g+1=(g+1)^{2}=0 \Rightarrow g \phi=-\phi$, some $\phi \neq 0, g^{p}=1 \Rightarrow$ contradiction. Hence $G$ is a 2-group. If $g \in G$ has order 4 , det $g= \pm 1$ $\Rightarrow \operatorname{det} g^{2}=1$. As above, this gives $\left(g^{2}+1\right)^{2}=0$, or $g^{4}+2 g^{2}+1=2+2 g^{2}=0$, or $g^{2}=-1$. The characteristic polynomial of $g$ is $g^{2}+2 g+\operatorname{det} g=-1+\operatorname{det} g+2 g=0$. $\operatorname{det} g=1 \Rightarrow g=0$, no go. If det $g=-1$, then $g=1$, contradicting $g^{2}=-1$. Hence $G$ has only elements of order 2 . Therefore $G$ is abelian elementary. $Z_{2} \oplus Z_{2} \oplus Z_{2} \notin G$, implying $G=1, Z_{2}$ or $Z_{2} \oplus Z_{2}$. Suppose $G=Z_{2} \oplus Z_{2}=\{1, x, y, x y\}$. $\operatorname{tr} x=\operatorname{tr} y$ $=\operatorname{tr} x y=-2$. If $N=1+x+y+x y$ were zero, taking traces implies $2-2-2-2=0$. Contradiction. Hence $\psi=N \psi_{0} \neq 0$, some $\psi_{0}$. Clearly $x \psi=\psi$. Now $x^{2}+2 x \pm 1=0$ and $x^{2}=1$, hence $x=0$ or $x=-1$. The first is nonsense, and the second implies $-\psi=\psi \Rightarrow \psi=0$. Contradiction! Hence only $G=1$ or $Z_{2}$ is permissible. Thus if $G$ reverses orientation, only $1, Z_{2}, Z_{4}, Z_{2} \oplus Z_{2}$ are permissible. Q.E.D.

Note. It is easy to see that each of these can occur.

Proposition 2.13. Let $H \triangleleft G, V=$ fix-point free complex representation of $H$. Then $C G \otimes_{C H} V$, a G-representation, is again $H$-free.

Proof. Trivial.

Using Proposition 2.13, we can construct free actions on $S^{2 n-1} \times S^{m}$ as follows: Let $1 \rightarrow H \rightarrow K \rightarrow 1$, exact, and suppose $H$ acts freely on $C^{a}$. Then $H$ acts freely on $C G \otimes_{H} C^{a} \approx C^{l a}, l=|G: H|$. We may suppose the action of $G$ is unitary. Therefore $G$ acts on $S^{2 a l-1}$ such that $H$ acts freely. If now $K$ acts freely on $S^{m}$, so $G$ acts freely on $S^{2 a l-1} \times S^{m}$ via $g(z, w)=(g z, \bar{g} w)$. This procedure immediately gives many free actions of nonabelian groups on $S^{n} \times S^{n}, n$ odd. In particular, any metacyclic group so acts (suitable $n$ ). We call these semiproduct actions.

EXAMPLE. $G$ of Type I: $1 \rightarrow Z_{p^{2}} \rightarrow G \rightarrow Z_{p} \rightarrow 1$ acts freely on $S^{2 p-1} \times S^{2 p-1}$. Therefore, the condition of Theorem $\mathrm{A}$ is necessary.

A nonsemiproduct action of $Z_{r}$ on $S^{2 n-1} \times S^{2 n-1}$, which is fix-point free, is as follows: Consider $S^{2 n-1} \subset C^{n}$, the unit sphere. Let $\zeta=e^{2 \pi i / r}$. If $z, w \in S^{2 n-1}$, set $T_{w}(z)=z+(\zeta-1)(z, w) w . T_{w}$ is unitary for fixed $w$. Indeed, $T_{w}=$ identity on $C w$, $T_{w}=$ multiplication by $\zeta$ on $C w$. Thus, $T_{w}^{r}=1$. Moreover, $T_{\zeta w}=T_{w}$.

Define $T: S^{2 n-1} \times S^{2 n-1} \rightarrow S^{2 n-1} \times S^{2 n-1}$ by $T(z, w)=\left(T_{w}(z), \zeta w\right)$. Clearly $T^{n}=1$, and $T$ is fix-point free. If $S(z, w)=(\zeta z, w)$, then $S T=T S, S^{r}=1$, and $\langle S, T\rangle \approx Z_{r} \oplus Z_{r}$ acts freely on $S^{2 n-1} \times S^{2 n-1}$.

In conclusion, we discuss the following 
ConjeCture II: If $G$ acts freely on $S^{n} \times S^{n}$, and $Z_{2} \oplus Z_{2} \subset Z(G)$, then $\left(Z_{2} \oplus Z_{2}\right)$ $\cap Z(G) \neq 1$. This has several points in its favor.

(1) It holds for any extension $G: 1 \rightarrow H \rightarrow G \stackrel{\pi}{\rightarrow} K \rightarrow 1$, where $H$ acts freely on $S^{a}, K$ acts freely on $S^{b}$. For let $L=Z_{2} \oplus Z_{2}=\{1, x, y, x y\}$. $H$ acts freely on $S^{a}$ implies $L \notin H$. If $L \cap H=1$, then $L \approx \pi(L) \subset K$. Contradiction. Therefore $H \cap L$ $=\{1, x\}$, say. By Milnor's Theorem $x \in Z(H)$ and is the only involution in $H$. As $\{1, x\}$ is characteristic in $H$, it is normal in $G$, hence $x \in Z(G)$. So Conjecture II holds in this case. In particular, it holds for semiproduct actions. A weaker statement, II', implies II :

(2) II': If $L=\{1, x, y, x y\} \subset G$, then $G=C(x) \cup C(y) \cup C(x y)$. Indeed, suppose II' holds. If $L \cap Z(G)=1$, we can fix $g, h \in G$ such that $g x=x g, g y \neq y g, h x \neq x h$, $h y=y h(\Rightarrow g h \neq 1)$. Consider $g h$. If $g h x=x g h$, then $h x=x h$; if $g h y=y g h$, then $h y=g h$; contradictions. Hence $g h x y=x y g h$, or $g y h x=y g x h$, implying $g^{-1} y g y$ $=x h x h^{-1}=u \neq 1$. Clearly $x u=u x, y u=u y$. And $u \notin L$ is evident. $x u=u x$ says that $x\left(x h x h^{-1}\right)=x h x h^{-1} x$, or $h x h^{-1} x=x h x h^{-1}$, implying $u^{2}=1$. Thus $L \oplus\langle u\rangle \approx Z_{2}$ $\oplus Z_{2} \oplus Z_{2} \subset G$. Contradiction. Thus $L \cap Z(G) \neq 1$, i.e. II' implies II.

(3) II holds if $G$ is an extension $1 \rightarrow G_{0} \rightarrow G \rightarrow Z_{2} \rightarrow 1$, such that $G_{0}$ is abelian. (Exercise.) Thus in particular, II holds for $S^{1} \times S^{1}$.

(4) II holds if $G$ is a 2-group. (Exercise.)

(5) II implies Milnor's Theorem. For if $G$ acts freely on $S^{n}, u \in G$, involution, then $Z_{2} \oplus Z_{2} \subset G \oplus G$ acting freely on $S^{n} \times S^{n}$. And $Z(G \oplus G)=Z(G) \oplus Z(G)$. Therefore II implies $Z_{2} \subset Z(G)$.

ReMARK. The group $G=\left(a, b: a^{8}=b^{2}=1, b^{-1} a b=a^{3}\right)$ acts freely on $S^{3} \times S^{3}$. $L=\left\langle a^{4}, b\right\rangle \approx Z_{2} \oplus Z_{2}$ is contained in $G$, but $L \not G$.

\section{BIBLIOGRAPHY}

1. R. D. Anderson, Zero-dimensional compact groups of homeomorphisms, Pacific J. Math. 7 (1957), 797-810.

2. L. E. J. Brouwer, Enumération des groupes finis de transformations topologiques du tore, C. R. Acad. Sci. Paris 168 (1919), 845.

3. H. Cartan and S. Eilenberg, Homological algebra, Princeton Univ. Press, Princeton, N. J., 1956.

4. P. E. Conner, On the action of a finite group on $S^{n} \times S^{n}$, Ann. of Math. (2) 66 (1957), 586-588.

5. A. Heller, A note on spaces with operators, Illinois J. Math. 3 (1959), 98-100.

6. Y. Kawada and J. Tate, On the Galois cohomology of unramified extensions of function fields in one variable, Amer. J. Math. 77 (1955), 197-217.

7. G. Lewis, The integral cohomology rings of groups of order $p^{3}$, Trans. Amer. Math. Soc. 132 (1968), 501-529.

8. L. N. Mann, Actions of elementary p-groups on $S^{n} \times S^{m}$, Michigan Math. J. 11 (1964), 47-51.

9. L. N. Mann and J. C. Su, Actions of elementary p-groups on manifolds, Trans. Amer. Math. Soc. 106 (1963), 115-126.

10. J. Milnor, Groups which act on $S^{n}$ without fixed points, Amer. J. Math. 79 (1957), 623-630. 
11. L. Rédei, Das schiefe Produkt in der Gruppentheorie, Comment. Math. Helv. 20 (1947), 225-264.

12. J. C. Su, Periodic transformations on the product of two spheres, Trans. Amer. Math. Soc. 112 (1964), 369-380.

13. R. G. Swan, A new method in fixed point theory, Comment. Math. Helv. 34 (1960), 1-16.

14. $\longrightarrow$ The p-period of a finite group, Illinois J. Math. 4 (1960), 341-346.

15. H. Zassenhaus, The theory of groups, Chelsea, New York, 1949.

University of California,

Berkeley, California 